\title{
Communication strategy of Web Portal Platishers
}

\author{
Wang Quan, Sheng Qizhi
}

The Northeastern University , Zhihui Street,Hunnan District,Shenyang City,Liaoning Province

wqwangquan00@126.com

Keywords: Platisher, self-media , Universal Mediation , Communication strategy.

\begin{abstract}
With the rapid development of information technology and digital technology, new media has become the main way for us to obtain information. The rapid development of social media has become an inevitable trend. With the promotion of mobile communication, big data analysis and the development of cloud computing, social media is rising rapidly. The concept of UGC allows a large number of self-media users to emerge. The major Internet companies rushed to launch self-media products, the WeChat public platform was complacent and self-appointed. The situation of a single big company was broken and it entered the era of publicity. The core competitiveness of self-media platforms is often manifested in the competition for high-quality content and the specification of platform content. The investigation of its communication strategy mainly includes six parts.
\end{abstract}

\section{Introduction}

With the rapid advancement of science and technology, information technology has achieved rapid development. In the era of web2.0, social media has rapidly emerged under the conditions of mobile communication, big data analysis, cloud computing, and sharing. In the era of web3.0, new and new media The convergence between new media is also in full swing. The content-oriented "media platform" and the "platisher" that is based on user relationships and content aggregation have become an effective way for the media industry to enhance its own competitiveness.

The portal site is an active practitioner of "platisher". Their platform-based media strategy has already begun in the PC Internet era. Diversified applications have emerged in the era of mobile Internet. There are competitions between the four types of portal websites in the domestic four major portals, namely, Sina, NetEase, Sohu and Tencent, each of which has its own advantages and disadvantages. The exploration of its communication strategy has its own characteristics, including six aspects.

\section{Cross-platform propagation to increase exposure}

The cross-platform media platform strategy is a commonly used method in the process of media integration and transformation, that is, through the horizontal development of cross-platform, expanding the dissemination channels of information, so as to realize the multi-channel flow of information. In order to adapt to changes in media usage by the audience, the media agency adopts cross-platform communication methods to increase the arrival rate and acceptance rate of media content and maintain its original competitiveness.

Cross-platform dissemination means cross-platform extension of information dissemination, which means expansion of information dissemination channels. This is often the only way to achieve transformation of various media including traditional media. However, most cross-platform content tends to be homogenous and highly similar. Therefore, in the process of cross-platform communication, it is necessary to change the form and focus of content dissemination according to the characteristics of the platform and achieve diversity in content marketing. With differentiated communication, build a cross-platform media matrix.

Coverage and arrival rate are the focus of traffic concerns in the mobile Internet era. "Qiehao" 
centralized Tencent, Tencent news software, daily Express client, Tencent video software, WeChat news plug-in and other Tencent business platform resources, covering over 800 million Internet users, Sina media open platform - "Sina Watch" use Sina News client Sina, Weibo, Sina and Sina mobile phone platform exposure resources, Sohu's self-media platform "Sohu" focus Sohu, mobile Sohu and Sohu News client three-terminal resources, Netease's NetEase media platform-"wangyihao" content is displayed centrally in NetEase News, and on the NetEase news client.

\section{Cross media integration, Media cooperation win-win}

In the process of media integration and transformation, different forms of media have different strategic choices. Cross-media integration is the internal logic of Internet technology development. It is an important mechanism for media organizations to adapt to users' needs and to achieve cross-media information output.

The cooperation between "Single Information" and the media such as Phoenix fully reflects the dual benefits of sharing advantages and traffic flow. One point of information provides data advantages while Phoenix provides its high quality content. This initiative has undoubtedly expanded the channels for the dissemination of information, brought about two-way traffic growth, realized the mutual benefit and win-win of both parties, and won more faithful users for the platform. For the audience, the common means that a large number of high-quality resources are introduced to each other. When the audience obtains high-quality content from the Phoenix Web, they can also enjoy a bit of information based on the interest of the user, in addition to satisfying user fragmentation time with interesting and interesting content. Consumer demand also meets the user's need for value enhancement in terms of valuable long-tailed content, in line with Maslow's hierarchy of needs.

On November 9, 2017, the Beijing News and Sina signed a comprehensive in-depth cooperation agreement, which stipulated that 26 media accounts under the Beijing News Group will be fully settled on the Sina Watchpoint platform. This is undoubtedly another important measure for platform-based media. The graphic and video content of the Beijing News will use Sina's watch platform to implement differentiated content recommendation and dissemination on the Sina news client, mobile Sina, Sina, and Weibo. At the same time, several boutique sections of the Beijing News will cooperate with Sina's light IP products in more depth.

\section{Enabling MCN to build content marketing ecology}

The popularity of user-generated content (UGC) does not mean that the threshold for content creation is reduced. Professional media and self-media work together to build a new content boundary. Users and content are reconstructed, and users and content are reconstructed. The dramatic increase in the number of content also poses challenges to the quality and dissemination of content. The excess content of the overall information brings more uncertainties to the information market. Therefore, to achieve content marketing, the most important thing is to create high-quality content. With the high degree of integration of content and channels and media, content marketing has become the model of cooperation that brand owners focus on.

The concept of Multi-Channel Network (MCN) begins with YouTube. Its essence is a product form of a multi-channel network. Combining PGC content can help creators amplify their advantages and make up for their disadvantages. It ensures the continuity of content through systematic operations. Output, and achieve stable commercial liquidity.

On November 29, 2017, the MCN Industry Development Conference and the Chengdu MCN Industrial Park launching ceremony were held in Chengdu. It marked the nation's first new Internet industry park that converged $\mathrm{MCN}$ organizations and concentrated media, creative and industrialized operations from media professionals. Chengdu MCN Industrial Park officially settled in Chengdu. The industrial park will provide funds, resources, platforms, policies, and other aspects 
of support for the commercialization of professional institutions and self-media accounts through centralized, standardized, and scaled operation methods, assisting them to create more suitable media market requirements. Content, thereby creating greater economic and social benefits.

In Tencent, the daily express newspaper that can issue the Qiehao platform content has achieved the content outsourcing production model; Erneng, Ivy Culture, WeMedia, Mars Culture, and Runaway Comics have become the first Tencent MCN strategic partners. Sina, on November 8, 2017, Weibo established a 3 billion fund to support MCN institutions to help content creators gain super exposure, Sina Watch will focus on supporting vertical areas MCN, video MCN, regional MCN, and Weibo strong The alliance will help MCN organizations build their high-quality account matrix, accelerate the pace of institutional development, and expand the influence of MCN. In terms of Netease, on November 7, 2017, NetEase's MCN function was put on the line, creating a three-dimensional account incubator system and opening access to MCN organizations in various vertical fields. By opening certain functional permissions to $\mathrm{MCN}$ institutions, it is convenient for them to centrally manage their sub-accounts, and help institutions build their high-quality account matrix through resource leaning and policy tilting to accelerate the pace of institutional development.

\section{Realization of commercialization of content to foster users}

Platform-based media often determine advertisement placement based on factors such as the amount of articles read, the degree of originality, and the quality of articles, and users receive revenue. After satisfying certain basic conditions, users can apply for the opening of original labels to obtain more subsidies, thereby encouraging The author produces more quality content.

Tencent Qiehao will automatically open the "share of revenue" function for Qiehao that have already passed trial operations and become officially operational. After being divided into operations, the creators will receive advertising from platform sharing and content sharing into revenue. In addition, to meet the 1 . Officially operated Qiehao, Qiehao opened WeChat content source synchronization, credit score 100 points, no demerit points record; 2. Formally operated for 30 days, issued a full 20 articles is recommended (the article recommended amount is greater than 0 ) The credit score is 100 points without any demerit points. The users of these two standards automatically activate the "Traffic Master" function, which means that the number of articles read is increased, the number of users covered increases, and the advertising revenue also increases.

NetEase's revenue is "platform revenue divided into", which is embodied in: 1. All access to the star system (up to a star and above) can open platform revenue authority. (Netease News copyright is excluded from the media.) Bonus revenue is related to account flow and article quality. 2. The Netease platform is divided into revenues mainly referring to the Netease index (readings, follow-ups, sharing amounts, etc.) and comprehensive assessment calculations. 3. Traffic plus policy. In combination with platform activities, specific subject content can obtain traffic markups, get higher traffic, and gain higher profits.

Sohuhao currently has no revenue function, which is not enough to attract creators. Sina Watch has opened bonus incentives. Sina's four-star and five-star authors who meet the following conditions (verticality $>70 \%$, long-term assignment $>30$ days, monthly photo or video release $>15$ articles, no violation in the past 30 days) will be eligible to participate in the bonus excitation.

It can thus be seen that the platform-based media are different in their strategy of using revenue to nurture users.

\section{Application of techniques such as algorithmic data}

Mobile cloud computing has greatly enhanced the processing capabilities of the Internet of Things; the positioning, transmission, and energy-saving technologies of the mobile Internet can all be used in the Internet of Things. Therefore, the future integration with the Internet of Things will be another important development direction of the mobile Internet. The development of pan-media 
under traditional media is obviously not smooth, which shows that the phenomenon of pan-media is also inseparable from the evolution of media technologies. The advancement of information technology has promoted the evolution of the new situation of information dissemination and disseminated high-quality content more accurately through data mining. A number of established Internet companies have launched self-media platforms. These platform-based media are obviously powerful expressions of media decentralization.

In order to allow Qiehao authors to more objectively understand their own performance and works, and to obtain more fair and stable recommendations and rewards at the same time, the Qiehao index was launched, from the user's favorite degree, content originality, account activity, content verticality, account number The five dimensions of health measure users. The Qiehao media platform allows content entrepreneurs to independently produce content through the openness of the content field, sort out the entire content industry from an industry perspective, and establish rules.

In order to optimize the rules for the calculation of the star rating system and allow the authors to create more standardized texts and understand the preferences of users, Netease launched the Netease index system. The Netease index reflects the overall performance of the account within a certain period of time and is a measure of the quality of the account. It mainly scores the value of the account generated by the account and the reader's evaluation of the account content, including five dimensions of health, originality, activity, professionalism, and interaction.

From an overall point of view, the application of data from various media publishing platforms has become more diversified. There are similarities and differences. In the final analysis, they are all for the promotion of the platform itself, regardless of the guidance of the operators. The role, or the realizing function and the social function, all improve the user experience to a certain extent, virtually cultivating the user's stickiness, and thus supporting the long-term development of the platform-based media.

\section{Strengthen core competitiveness}

The core competitiveness of the media is the media's resources and ability to win the audience, occupy the market, obtain the best economic and social benefits, and maintain a unique competitive advantage among many competitors. A successful business model determines whether a company can reasonably integrate its corporate resources and bring its core competencies into play.

For example, Qiehao's advantage lies in its strong data flow support behind it. This is other self-media platforms, including Ali's UC hao are unmatched. Tencent, as one of the largest Internet service providers in China and one of the Internet companies with the most service users, has established a stable user base through instant messaging software QQ, expanding other businesses under this advantage, and using Internet value-added services, such as game recharge, each The VIP recharging was profitable, which provided Qiehao with sufficient funds and flow support.

Baidu has obvious advantages in the field of search engines. Baidu's "Hundred Houses" has amassed a variety of application styles such as information retrieval, information provision, public discussion, navigation services, community services, mobile services, entertainment games, and software tools. The functional structure encompasses most of the users' needs and becomes a representative, comprehensive platform-based media. The advantages of NetEase are reflected in its content. The Sina Watch Platform is based on the good brand awareness established by Weibo and the Sina Portal.

But no matter what, user-oriented and market-oriented, technology-driven, platform-based development trends determine that platform-based media can only stand out from their competitors by enhancing their core competitiveness.

\section{References}

[1] Xu Tongwen, "Media Platforms and Platform Media: Approaches to Media Transformation in the Mobile Internet Era" [J], Journalism, 2015, Issue 13, 50-52. 
[2] Liang Ziying, "Data application model of self-published media platforms: A comparative study of the WeChat public platform and Today's headline" [J], Information Technology Search, 20171674-6708 (2017) 188-0052-02.

[3] Zhai Zhicheng, Development Status and Development Trend of Mobile Internet [J], Mobile Communications, 2008, 01 (09): 22-24.

[4] Lu Gaofeng, "From the Media Entering the Public" and "The Times" [J], "Young reporters", December 2016, Peak Observations, p. 118. 